\title{
Redo surgical biological valve replacement: Gone with the wind?
}

\author{
Filip P. Casselman, MD, PhD, FETCS
}

\author{
From the Department of Cardiovascular and Thoracic Surgery, OLV Clinic, Aalst, Belgium. \\ Disclosures: Dr F. Casselman is a consultant for Edwards Lifesciences and Medtronic \\ Received for publication April 3, 2018; accepted for publication April 6, 2018; available ahead of print May 17, \\ 2018. \\ Address for reprints: Filip P. Casselman, MD, PhD, FETCS, Department of Cardiovascular and Thoracic Surgery, \\ OLV Clinic, Moorselbaan 164, 9300 Aalst, Belgium (E-mail: Filip.Casselman@olvz-aalst.be). \\ J Thorac Cardiovasc Surg 2018;156:1355-6 \\ $0022-5223 / \$ 36.00$ \\ Copyright $(C) 2018$ by The American Association for Thoracic Surgery \\ https://doi.org/10.1016/j.jtcvs.2018.04.020
}

Currently, there are 3 major therapies available for aortic valve replacement (AVR): they are in chronological order of appearance in the surgical armamentarium AVR through sternotomy, minimally invasive (mini)-AVR, and transarterial percutaneous valve replacement (TAVR). The evolution toward mini-AVR and TAVR requires mastering catheter-based skills. As such, the "classical" surgeon who also performs mini-AVR and TAVR becomes what I would call a "surgical interventionalist" (as opposed to a cardiological interventionalist). This applies to AVR and currently to a lesser extent also to mitral valve procedures (MVR).

Surgical interventionalists are the only physicians present in a hospital who perform all 3 procedures. Therefore, these physicians are in the pole position to decide what is the best AVR treatment for a given patient. To make this decision, a patient is usually (and should always be) discussed in a multidisciplinary meeting (heart team). Scientific evidence is available to a certain extent to guide physicians. In addition, various risk scores are available, although all have their shortcomings. The proposed therapy for a given patient should be a well-balanced and careful decision based on available evidence. This evidence is not overwhelming in reoperative settings, and in the everyday heart team meeting, we regularly observe that our cardiological interventional colleagues are easily in favor of TAVR whenever a reinterventional procedure is considered.

The current paper by Stulak and colleagues ${ }^{1}$ in this issue of the Journal provides us additional information on reoperative aortic and mitral biological valve replacement outcomes. The combined operative mortality was $8 \%$ and risk factors were increasing New York Heart Association (NYHA) functional class and previous coronary artery bypass grafting (CABG). The absence of NYHA class III/ IV or previous CABG resulted in a combined AVR-MVR reoperative mortality of $4 \%$. The exact individual AVR versus MVR cohort mortality is not provided, but we know from previous studies that isolated reoperative aortic interventions. $^{2}$

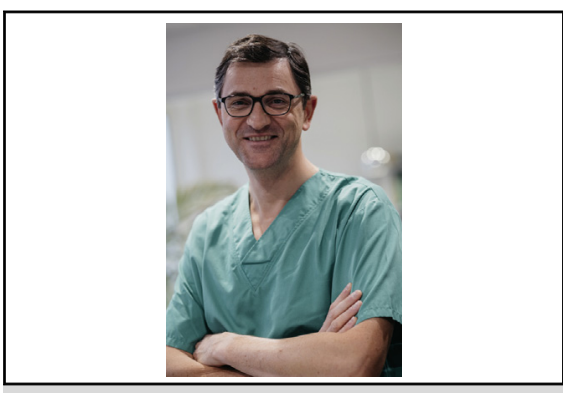

Filip P. Casselman, MD, PhD, FETCS

Central Message

Surgical interventionalists are in the pole position to balance institutional results and risk factors to decide between an open, minimally invasive, or percutaneous approach for a given patient with valve disease.

See Article page 1380 .

valve replacement mortality almost equals primary

These data provide strong evidence over a 20-year period that reoperative procedures do come at a relatively low risk and these results should be taken into account whenever considering a valve reintervention for a given patient. Whenever negative prognostic factors are present (NYHA III/IV or previous CABG), the authors advise lower-risk procedures (and suggest TAVR). Unfortunately, the current manuscript does not detail whether a TAVR approach would have resulted in a lower-risk procedure in such a population.

The authors detected a high proportion of NYHA functional class III and IV in their study population. They argue correctly to follow up patients more closely and potentially refer these patients earlier for therapy. This is also an argument for surgical interventionalists to be more involved in the follow-up of patients in valve clinics. Earlier detection and referral should lower periprocedural mortality.

Another consideration in the treatment choice is the increasing proportion of patient-prosthesis mismatch associated with valve-in-valve procedures. ${ }^{3}$ This condition is known to influence outcomes negatively and is certainly an important contributing element in the decision-making process regarding the optimal treatment and symptomatic relief for a given patient. 
Finally, every surgical interventionalist should be perfectly aware of his or her own institutional results of the 3 valve therapies available. Only then a balanced decision can be made.

This editorial is by no means a plea against TAVR! TAVR is a great therapy that has helped numerous patients who were otherwise at greater surgical risk. TAVR has proven its merits and will certainly continue to do so in the future. Newer indications will arise and should be balanced against available therapies. In the meantime, clear involvement of surgical interventionalists in the follow-up, referral, and decision-making is imperative. Knowledge of institutional results, risk factors, and outcome determinants of AVR, mini-AVR, and TAVR should help to make a balanced decision in every patient. Clearly, surgical redo AVR is a fully available therapy in this regard and should not be neglected!
As demonstrated in the current paper by Stulak and colleagues, results are excellent and should play as a benchmark in patient selection for one procedure or another. Redo surgical biological valve replacement is certainly not gone with the wind!

\section{References}

1. Stulak JM, Tchantchaleishvili V, Daly RC, Eleid MF, Greason KL, Dearani JA, et al. Conventional redo biological valve replacement over 20 years: surgical benchmarks should guide patient selection for transcatheter valve-in-valve therapy. J Thorac Cardiovasc Surg. 2018;156:1380-90.

2. Davierwala P, Borger MA, David TE, Rao V, Maganti M, Yau TM. Reoperation is not an independent predictor of mortality during aortic valve surgery. $J$ Thorac Cardiovasc Surg. 2006;131:329-35.

3. Faerber G, Schleger S, Diab M, Breuer M, Figulla HR, Eichinger WB, et al. Valve-in-valve transcatheter aortic valve implantation: the new playground for prosthesis-patient mismatch. J Intervent Cardiol. 2014;27: 287-92. 